\title{
Origins of Life Research Does Not Rest on a Mistake
}

\author{
BRIAN KNAB \\ University of Texas at Austin
}

\begin{abstract}
I defend origins of life research against an argument, given by Roger White in 2007, that it rests on a mistake. I show how the Bayesian machinery can illuminate the rational search for alternative explanations of inexplicable, improbable data, and in particular how it can illuminate the rational search for a secular explanation of the origins of life and of the fine-tuning of the universe.
\end{abstract}

\section{Introduction}

Self-replicating molecules are complex. Thus even if each individual step in their natural construction was probable, the overall probability of randomly constructing a complete self-replicator could be minuscule. ${ }^{1}$ And given this, some have been unable to rest comfortably with the idea that self-replicators and thus life arose by chance; they contend that there must be some further explanation available. ${ }^{2}$ Some are right now in laboratories searching for that further explanation.

Roger White-in his 2007 paper, "Does Origins of Life Research Rest on a Mistake?"- argues that this search is unmotivated. He does not dispute the claim that it was improbable that life should have arisen by chance. Instead he argues: the fact that it was improbable is no reason to think that it didn't.

Contact: Brian Knab <brian.knab@utexas.edu>

1. In order for this to be true, by a 'randomly generated' step, we must mean a step which is probabilistically independent of the prior steps. Also, it is important to note that-granting the random construction of a particular self-replicator along a particular path was improbable-it does not follow that the random construction of self-replicators in general was improbable. If there were many possible self-replicators, or if, for any particular self replicator, there were many ways to construct that self-replicator, then self-replication would not be an improbable outcome. (Thanks to an anonymous reviewer for this latter point.) As we'll see, I am going to grant that self-replication is, in general, improbable. The question I am concerned with is: granting self-replication is improbable, what is the rational reaction to this improbability?

2. Roger White (2007 460) attributes this view to origins of life scientists J. D. Bernal, Manfred Eigen, Christian De Duve, and Richard Dawkins. 
Here, I defend the searchers against White. His argument overgeneralizes. It entails that it is always irrational to search for an explanation of currently inexplicable empirical data. But this is not always irrational.

Further, I argue that it often is reasonable to search for alternative explanations of your data, upon discovering that it is very improbable that that data should have arisen by chance. It is reasonable to do so when you are unsure about the conditions under which your data arose. In recognizing this, we can explain both why we ought to search for an explanation of the origin of life, but also I think, why we ought to search for an explanation in the related case of the fine-tuning of the universe.

\section{Preliminaries}

Allow me some preliminaries. First, I will refer to the hypothesis that an event happened by chance as the chance hypothesis. What I mean is: the hypothesis that an event happened by chance, under some (often implicit) dominant probability distribution over the space of events. Suppose that you have two dice and you roll a double six. The chance hypothesis (in normal contexts) is the hypothesis that the dice are fair. ${ }^{3}$

Second, I will assume that a self-replicating molecule, and thus life, was an improbable outcome under the relevant chance hypothesis. De Duve (1991), for example, claims that the probability of life's arising by chance was less than 1 in 1o raised to the 3ooth power. Hoyle and Wickramasinghe (1981) calculate it to be around $I$ in 10 raised to the 40 ,oooth power. ${ }^{4}$ Very roughly, this is the probability that self-replicating molecules should arise through the haphazard mixing of the non-self-replicating material present on early Earth. Without getting into the details, it's hard to evaluate these claims. But luckily, we needn't; we're just going to grant them.

Third, the mere fact that an event occurred which was improbable, according to some hypothesis, is not evidence that that hypothesis is false. I once read that every time you shuffle a deck of cards, and deal a hand in a game of Spades,

3. The chance hypothesis need not, however, be uniform over the space of atomic eventsimagine that we confront a pair of trapezoidal six-sided dice, for example. Here the dominant distribution will roughly be the one which assigns a probability to each face equal to the ratio of the surface area of that face to the total surface area of each die. In this case, a statement like 'you rolled snake eyes by chance' remains perfectly intelligible, even though the probability distribution over each face is not uniform. Of course there are tricky questions here-why prefer the dominant probability model to others? And how do dominant probability models become dominant in the first place? I'm going to try to avoid saying anything here that commits me to an answer to any of these larger questions.

4. Both of the de Duve (1991) and Hoyle and Wickramasinghe (1981) references are from White's 2007) paper, and he attributes them to Fry (2000). 
say, it is likely that that particular hand has never been generated in the entire history of card play. This, because the number of distinct ways to distribute 52 cards among four players is $1.25 \times 10^{28}$, which is about 1 billion times greater than the number of grains of sand on Earth. ${ }^{5}$ Nevertheless, most hands are not evidence against the hypothesis that the cards were randomly shuffled.

This, however, raises a question: when is the occurrence of an event, improbable according to some hypothesis, evidence against that hypothesis? The answer, according to White and which we will rely on, is given by the following theorem:

$$
P(C \mid E)<P(C) \quad \text { if and only if } \quad P(E \mid \neg C)>P(E \mid C)
$$

In words: if $C$ is the hypothesis that the cards were randomly shuffled, and we confront some evidence $E$-i.e., a particular hand-that hand is evidence against the random-shuffling hypothesis if and only if that hand is more likely given the cards were not shuffled randomly, than given they were.

In the following, we will not be directly concerned with (1), but instead with a necessary condition that follows on (1), namely, that if $\left\{B_{1}, \ldots B_{n}\right\}$ forms a partition of $\neg C$-that is, if $\neg C$ is equivalent to the disjunction $B_{1} \vee B_{2} \vee \ldots \vee B_{n}$, and $B_{1}, \ldots, B_{n}$ are mutually exclusive-then

$$
\begin{aligned}
& P(C \mid E)<P(C) \quad \text { only if } \\
& P(E \mid C)<P\left(E \mid B_{1}\right) \text { or } P(E \mid C)<P\left(E \mid B_{2}\right) \text { or } \ldots \text { or } P(E \mid C)<P\left(E \mid B_{n}\right)^{6}
\end{aligned}
$$

Or again in words: Suppose I know that the cards were either shuffled randomly or that either Bob stacked the deck or Bill did. Then I get evidence the cards were not shuffled randomly only if my hand is more likely given Bob stacked the deck, or given Bill did.

\section{White's Example}

Preliminaries discharged, I turn now to White's argument; it begins with an example. 2012).

5. According to Krulwich's estimate of the number of grains of sand on Earth (Krulwich

6. Suppose that for all $i, P(E \mid C) \geq P\left(E \mid B_{i}\right)$. Then we have

$$
\begin{aligned}
P(E \mid \neg C) & =\sum_{i} P\left(B_{i} \& E \mid \neg C\right)=\sum_{i} P\left(E \mid B_{i} \& \neg C\right) \cdot P\left(B_{i} \mid \neg C\right)=\sum_{i} P\left(E \mid B_{i}\right) \cdot P\left(B_{i} \mid \neg C\right) \\
& \leq \sum_{i} P(E \mid C) \cdot P\left(B_{i} \mid \neg C\right)=P(E \mid C) \cdot \sum_{i} P\left(B_{i} \mid \neg C\right)=P(E \mid C) \cdot P(\neg C \mid \neg C)=P(E \mid C)
\end{aligned}
$$

Hence by theorem $(1), P(C \mid E) \geq P(C)$ 
Suppose we are headed to the English seaside. And consider three things we might confront upon arrival:

Pebble- $R$ The pebbles on the beach are scattered haphazardly over the beach.

Pebble-N The pebbles "cover the beach in descending order of size toward the shoreline."

Pebble-I The pebbles are "arranged to form a stick figure with a smile on its face" (White 2007 455). ${ }^{7}$

Each of these patterns could arise by chance-that is, via the standard physical processes by which pebbles come to lie on beaches, whatever they are. And let us suppose that each of our three patterns is equally likely under this chance hypothesis. (Nothing turns on this.)

White notices that if we confronted Pebble-N or Pebble-I, we would be suspicious of the chance hypothesis-we would not think that the pebbles just happened to be ordered by size, or arranged into the shape of a stick figure, by a mechanism randomly tossing pebbles onto the beach.

And according to ( 1 ), we get evidence that a pebble arrangement did not arise by chance only if it was more likely given it did not arise by chance than given it did.

Now, for White, to say that an arrangement did not arise by chance is just to say that the process which generated it was biased in some way. And Pebble-N and Pebble-I helpfully exemplify the two distinctive ways White thinks a process might be biased. He writes,

A process such as pebble arranging is intentionally biased if certain elementary possible outcomes are more likely than others due to the purposeful action of some agent ... A process is non-intentionally biased if this biasing is [due to] ... , say, the impersonal laws of nature together with properties of matter and the structure of physical mechanisms. 2007 462)

Call our evidence $E$ and the chance hypothesis $C$. Let $I$ be the hypothesis of intentional biasing, and NI be the hypothesis of non-intentional biasing. Then, by the necessary condition specified in (3) above, we have

$$
P(C \mid E)<P(C) \text { only if } P(E \mid I)>P(E \mid C) \text { or } P(E \mid N I)>P(E \mid C)
$$

Note that were we to confront Pebble-I or Pebble-N, this necessary condition would be satisfied. White writes about Pebble-N,

7. Pebble- $R$ for 'random', Pebble- $N$ for 'non-intentionally biased' and Pebble-I for 'intentionally biased.' 
That [the pebbles] should be arranged roughly in order of size seems more likely on the assumption that the process shuffling the pebbles was non-intentionally biased, than if they just fell on the beach by chance ... [If] attributes of the pebbles interact with the physical laws ... simple correlations between physical parameters such as size and location are the kind of phenomena we should expect to find. 2007:462)

And about Pebble-I,

The stick figure is one of a small class of interesting patterns, and if an agent was to go to the trouble of influencing the way the stones are arranged, there is a good chance she would arrange them in an interesting way. (2007, 462)

If we confronted Pebble- $R$, however, (4) would not be satisfied. Given an agent arranged the pebbles, the particular haphazard scatter we witness would not be likely. Further there aren't any obvious correlations between the physical attributes of the pebbles, so neither is the arrangement likely given non-intentional biasing. Intentional and non-intentional biasing thus make the haphazard arrangement just as unlikely as the chance hypothesis. And therefore a haphazard scatter does not call the chance hypothesis into question.

I think that this is an illuminating answer to the question of why certain arrangements of pebbles at the English seaside might cause us to suspect the chance hypothesis, while other, perhaps equally unlikely, arrangements under that hypothesis would not.

White now wants to generalize to the origins of life. He wonders: are moleculesarranged into self-replicating structures-akin to pebbles scattered haphazardly across a beach, or are they instead like pebbles arranged in order of their size, or perhaps, like pebbles arranged into the shape of a stick figure?

\section{White's Argument}

The rest of White's argument is now straightforward: First, he notes that intentional biasing in favor of life is not a hypothesis taken seriously by most scientists. Thus our evidence calls the chance hypothesis into question only if life was more likely given non-intentional biasing than it was given chance. But, White argues, life was not more likely given non-intentional biasing than given chance. And hence we have no reason to doubt the chance hypothesis.

That argument is valid, and I won't dispute the claim that scientists do not take the intentional biasing hypothesis seriously. But why should we agree with White that-given the process was non-intentionally biased—life would not be a likely outcome, or at least more likely than it would be given mere chance? White writes, 
I can't imagine why anyone would think [that life is more likely, given the process by which it arose was non-intentionally biased]. While there is at least room to argue that a rational agent is likely to influence [the process] in order to allow for the evolution of life, to suppose that impersonal physical laws are likely to constrain [it] in this way can only be based on a confused anthropomorphism ... Even if the value we attach to life is something objective, whether it be moral or aesthetic, or whatever, it could only conceivably have influence on the behavior of an agent. Blind physical laws are no more naturally drawn toward states of affairs with value than blind chance is. 2007:466)

And later,

Are self-replicating, life producing molecules more likely to appear on [the assumption that the process by which life arose was non-intentionally biased]? ... What makes certain molecular configurations stand out from the multitude of possibilities seems to be that they are capable of developing into something which strikes us as rather marvelous, namely a world of living creatures. But there is no conceivable reason that blind forces of nature or physical attributes should be biased toward the marvelous. 2007. 467)

In conclusion, White writes, "unless we suspect that life arose on purpose, we should be quite content ... in seeing life as an extremely improbable, 'happy accident'" 2007: 467).

This, then, is the mistake that origins of life research rests upon: scientists, searching in their laboratories, mistakenly believe that they have good reason to suppose the chance hypothesis is false, and hence that some other non-chancy explanation is available. But their data does not disconfirm the chance hypothesis, and hence they have no reason to search for an alternative explanation.

\section{Counter}

Despite White's argument, I think the searchers are justified in seeking an alternative explanation of life's origin; I turn now to their defense.

First, consider a case. Suppose we filled 1, ooo paper bags full of the molecules thought to be plentiful on early Earth, and we shook them up. And suppose that when we dumped out the bags, 90 percent of them yielded self-replicators. It could happen that 90 percent of our bags yield self-replicators even if no bias exists, and thus the probability of generating a self-replicator is minuscule. Nevertheless, I contend, it would not be reasonable to believe this chance hypothesis in the face of our data. We should not be content, in other words, to see our 
paper bag self-replicators as an "extremely improbable, "happy accident."' And this would be true, I think, even if (i) we are unwilling to countenance intentional intervention as a plausible explanation of the outcome, and (ii) we cannot at present tell any plausible story which would explain why "the impersonal laws of nature together with properties of physical matter and the structure of physical mechanisms" should favor self-replicators.

For a more realistic case, but with a similar upshot, suppose that we discovered that the rate of heart disease in San Diego was twenty times higher than in other cities of similar size and demographics. That would be strong evidence that something in San Diego is amplifying (or something in the other similar cities is suppressing) heart disease. And again it would not be reasonable, in the face of that data, to conclude that the amplified rate of heart disease in San Diego is just a sad accident-that the rate of heart disease in San Diego just, by chance, happens to be twenty times higher than in similar cities. And this would be true even though (i) agential intervention is not a plausible explanation of amplified rates of heart disease in San Diego, and (ii) we have no antecedent reason to believe that blind physical laws and forces of nature have it out for San Diegans.

So, data can call a chance hypothesis into question, or render it unreasonable to believe, even when (i) agential intervention is not a plausible explanation of that data, and (ii) we cannot presently tell a story about why blind physical laws and physical forces should favor that data. And thus, the fact that we cannot presently offer "any conceivable reason that blind physical forces of nature or physical attributes should be biased toward the marvelous" does not entail that the existence of the marvelous-i.e., the presence of life on Earth-fails to call into question the chance hypothesis, nor does it require that we view the marvelous as a happy accident. This is true even if, as with our bags of molecules and our San Diegans, we're committed to the view that intentional biasing is not a plausible explanation of the outcome.

\section{Diagnosis}

But how can this be? Didn't White rely on a theorem of the probability calculus? Am I denying math or Bayesianism or both?

White did rely on a theorem. And I am neither denying Bayesianism, nor math. Here is the theorem again: Where $\left\{B_{1}, \ldots, B_{n}\right\}$ forms a partition of $\neg C$,

$$
\begin{aligned}
& P(C \mid E)<P(C) \quad \text { only if } \\
& P(E \mid C)<P\left(E \mid B_{1}\right) \text { or } P(E \mid C)<P\left(E \mid B_{2}\right) \text { or } \ldots \text { or } P(E \mid C)<P\left(E \mid B_{n}\right)
\end{aligned}
$$

To see why what I've said is consistent with this theorem, consider another example: suppose that a coin has been discovered, deep in a tectonic fissure, 
clearly formed by blind physical laws and forces of nature. And now suppose that I hand you a coin, and I tell you: this is either a standard, fair coin, or it's the tectonic coin. (The tectonic coin is, of course, indistinguishable from a standard coin.) We'll flip it, and see how our subjective probability functions evolve.

We have no reason to think that blind physical laws and forces of nature should favor heads over tails or vice versa, and so, let's suppose, if we knew the coin was tectonic, we would adopt a uniform distribution over every possible weighting. ${ }^{8}$

Now suppose we flip the coin twice, and it comes up heads both times. Notice that the chance hypothesis is disconfirmed by this evidence. ${ }^{9}$ And because it's a theorem, you must therefore satisfy the necessary condition (3). In the case of the tectonic coin you do satisfy (3) simply because double heads is more likely given the coin is tectonic than it is given it's fair.

But the moral is: you can think that there are only two alternatives-a chance hypothesis versus a blind physical process that is just as likely to be biased against, as in favor of, each particular elementary outcome-and yet in witnessing those elementary outcomes receive evidence that disconfirms the chance hypothesis.

Perhaps, returning to our earlier examples, when we started shaking our bags of molecules, we thought that blind physical processes were just as likely to favor as to disfavor self-replicators. Nevertheless, if the chance hypothesis is that each step in a molecular construction occurs randomly, witnessing enough selfreplicators will disconfirm that hypothesis. The same goes for our San Diegans; perhaps prior to witnessing any data, we thought blind physical forces were just as likely to suppress as to amplify the rate of heart disease in San Diego. Nevertheless, if the chance hypothesis is that, in fact, nothing is amplifying or suppressing heart disease in San Diego, witnessing enough heart disease will again disconfirm that hypothesis.

The illicit inference that White draws is that because we have no reason to think that a blind physical mechanism would favor the marvelous over the nonmarvelous, that therefore our total marvelous evidence was just as likely to be produced by chance as by that blind physical mechanism. The latter doesn't follow from the former.

8. Setting Bertrand's paradox aside at our peril.

9.

$$
\begin{aligned}
& P(H H \mid \text { Fair })=\frac{1}{4} \\
& P(H H \mid \text { Tectonic })=\int_{0}^{1} P(H H \mid w) \cdot P(w) d w=\int_{0}^{1} w^{2} \cdot 1 d w=\frac{1}{3}
\end{aligned}
$$

Hence by theorem (1), Fair is disconfirmed. 


\section{Responses}

I see two possible responses available to White. First, there is something a bit strange about our tectonic coin, which I have passed over thus far in silence. Perhaps you noticed that had we flipped our coin only once, then the chance hypothesis would not have been disconfirmed by a heads outcome. ${ }^{10}$ And so White might contend that our actual evidence, in the case of the origins of life, is not like double heads, but like a single heads.

But first, to stave off any confusion, it is worth pointing out that sometimes a chance hypothesis can be disconfirmed by a single data point. Suppose that I hand you a coin which is either weighted to come up tails with probability .75, or it is the tectonic coin. Suppose we flip the coin once, and it comes up heads. If the chance hypothesis is that the coin is the tails-weighted coin, then that hypothesis is disconfirmed by a single heads outcome.

Second, White could perhaps contend that our evidence is like a single heads outcome, simply in that it is equally likely under the chance and non-intentional bias hypotheses. But my point is that he must argue for this conclusion. White infers from the fact that "there is no conceivable reason that blind forces of nature or physical attributes should be biased toward the marvelous" that therefore our evidence is equally likely under the chance and non-intentional bias hypotheses. But, again, this doesn't follow. We can suppose the chance and the blind physical forces hypotheses do assign equal probability to the generation of a single selfreplicator. Nevertheless, if our data has a certain composition-if it's akin to a tectonic coin coming up heads twice-then the chance hypothesis will yet be disconfirmed by our data.

So in order for White to successfully argue that the chance hypothesis is not disconfirmed, he has to (i) tell us what the chance hypothesis is, (ii) argue that it assigns to self-replicators a probability equal to or greater than the probability assigned by the hypothesis of unknown bias, and finally (iii) argue that our data is not composed in such a way that it nevertheless disconfirms the chance hypothesis. And that strikes me as a tall order.

The second response available to White is this: he can argue that while perhaps he hasn't definitively shown that our data fails to disconfirm the chance hypothesis, neither have origins of life researchers definitively shown that it does disconfirm the chance hypothesis.

10.

$$
\begin{aligned}
& P(H \mid \text { Fair })=\frac{1}{2} \\
& P(H \mid \text { Tectonic })=\int_{0}^{1} w \quad d w=\frac{1}{2}
\end{aligned}
$$


Allow me, then, to offer a toy model to the origins of life researchers, which I think can plausibly be extended to a probabilistic model of the origins of life, and according to which a self-replicator does disconfirm the chance hypothesis.

Imagine Nature sitting down at a 26-letter typewriter, and think of every key on the typewriter as a molecule present on early Earth. A priori, it seems that Nature is just as likely to be biased in favor of typing the letter $a$ as she is to be biased in favor of typing any letter, let's suppose, and this induces a uniform prior distribution over the space of possible bias hypotheses. ${ }^{11}$ Let us suppose further that the chance hypothesis is that every key is equally likely to be struck. Nature then begins to type.

Certain strings, of course, would do nothing to disconfirm the chance hypothesis, e.g.,

$$
\text { alkwersbn } n^{12}
$$

But certain other fairly mundane strings would disconfirm the chance hypothesis, e.g.,

$$
\text { alkklaaal }^{13}
$$

What is the difference between alkwersbn and alkklaaal? Repetition. Repetition is indicative of bias in favor of the repeated outcomes, and is also evidence that not every outcome is equally likely; i.e., it is evidence that the chance hypothesis is false.

11. Officially, this will induce a Dirichlet prior with 25 concentration parameters all equal to, say, 1 .

12.

$$
\begin{aligned}
P(\text { alkwersbn } \mid \text { Chance }) & =\frac{1}{26^{9}} \approx 1.8 \times 10^{-13} \\
P(\text { alkwersbn } \mid \text { Unknown Bias }) & =\int_{W} P(\text { alkwersbn\&W }) d W \\
& =P(\text { alkwersbn } \mid W) \cdot P(W) d W \\
& =\int_{W} w_{a} w_{l} w_{k} w_{w} w_{e} w_{r} w_{s} w_{b} w_{n} \cdot \Gamma(26) d W \\
& =\frac{\Gamma(26)}{\Gamma(35)} \approx 5 \times 10^{-14}
\end{aligned}
$$

Hence the chance hypothesis is confirmed.

13.

$$
\begin{aligned}
P(\text { alklaaal } \mid \text { Chance }) & =\frac{1}{26^{9}} \approx 1.8 \times 10^{-13} \\
P(\text { alklaaal } \mid \text { Unknown Bias }) & =\int_{W} w_{a}^{4} w_{k}^{2} w_{l}^{3} \cdot \Gamma(26) d W \\
& =\frac{\Gamma(26) \cdot \Gamma(5) \cdot \Gamma(4) \cdot \Gamma(3)}{\Gamma(35)} \approx 1.5 \times 10^{-11}
\end{aligned}
$$

Hence the chance hypothesis is disconfirmed. 
Thus, to complete the argument, we can simply note that, because self-replicating molecules consist of repeated molecular substructures, those molecules disconfirm the chance hypothesis. That, in other words, is a reason to think that selfreplicating molecules are more likely to result via blind forces of nature than via chance, and it is a reason which simply rests on a prior uniform distribution over the space of bias hypotheses, and not on a confused anthropomorphism.

Now, to be fair, White does anticipate something like the story I am telling here, and he writes in response that, in fact, "the picture given by de Duve and others is [that] ... the molecular parts required to make up the replication machinery come in various sizes and structures, and they are not arranged in anything like a simple repetitive pattern" (2007, 473). In other words, self-replicating molecules are more like the string alkwersbn than they are like the string alkklaaal.

But that strikes me as frankly incredible, and White does not give us a specific reference. You cannot look at the double helix of DNA, which is a (two-meter long, in humans, ) molecule consisting of a sugar, a phosphate, and four nucleobases repeated over and over and over, and not see a simple repetitive pattern. It is as though Nature sat down at the typewriter described above, and typed

$$
\text { AAAGTCTGACAAGCTACGCGGG .. }{ }^{14}
$$

And that, for the same reason as the string alkklaaal, will certainly disconfirm the chance hypothesis.

Of course, this model is not a perfect probabilistic representation of the origins of life. But that's not the point. The point is only that its general contours look right. And on any model which shares those general contours, a self-replicating molecule will disconfirm the chance hypothesis.

\section{Fine Tuning}

We have thus far been considering the molecular origins of life; I now want to turn to another case White discusses that is related, and which is I think more amenable to his argument: the apparent fine-tuning of the universe.

Some have noticed, for example, that had the rate of expansion of the early universe been slightly different, then a stable universe capable of producing and sustaining life would not have existed. ${ }^{15}$ They have inferred from this that it was extremely improbable that a universe like ours should exist. And here, we have access to only a single data point-i.e., a single universe. Thus we cannot rely, as

14. $A, G, T$, and $C$, of course, for the nucleobases adenine, guanine, thymine, and cytosine, which compose the nucelotides that compose DNA.

15. See Hawking: "If the rate of expansion one second after the Big Bang had been smaller by even one part in one hundred thousand million million, the universe would have re-collapsed before it ever reached its present state" 1996 156). 
in the case of the molecular origins of life, on the presence of repetition to show that the chance hypothesis is disconfirmed.

Though that last paragraph does not establish it, let us grant that, under the chance hypothesis, it was unlikely that our life-supporting universe should exist. If we're unwilling to countenance agential intervention as an explanation, must we, by White's argument, accept that a fine-tuned universe was an extremely improbable 'happy accident'?

The answer is 'no,' and this brings me to a second, much simpler, objection I want to raise to White's overall argument.

Suppose White is right that our evidence does not disconfirm the hypothesis that life and the universe arose by chance. And consider one last case. Suppose that in addition to a coin, our tectonic fissure had yielded a die with, say, one quintillion sides. We've analyzed the die in the lab, and have discovered that the die is either fair, or it is weighted to only come up 1 or to only come up 2 or ... or to only come up 1 quintillion. Along with White, we dub the hypothesis that the die is fair the 'chance hypothesis.'

Because we have no reason to favor any one of these hypotheses, it seems reasonable to adopt a uniform distribution over each weighting hypothesis. That is, given what I've said so far, we should think it equally likely that the die is fair as that the die is weighted to only come up in one particular way. Now, suppose we roll the die only once, and it comes up, say, 42. What should we think? Should we think that 42 was just an extremely improbable 'happy accident'?

We should not! If we're Bayesians, upon witnessing 42 , we should think it 1 quintillion times more likely that the die is weighted to come up only 42, than that it is fair. ${ }^{16}$

Put simply: the fact that the chance hypothesis is not disconfirmed by our evidence does not entail that we should think the chance hypothesis is true, and that therefore the outcome was a happy accident. The important question, if we're Bayesians, is not 'was the chance hypothesis disconfirmed?' but instead 'is the chance hypothesis, in light of our data, at all likely to be true?'

I think that our epistemic situation, with respect to the origins of life and

16.

$P($ Fair|42)

$$
\begin{aligned}
P(\text { Fair } \mid 42) & =\frac{P(42 \mid \text { fair }) \cdot P(\text { Fair })}{P(42 \mid \text { Fair }) \cdot P(\text { Fair })+P(42 \mid \text { Only } 42) \cdot P(\text { Only } 42)} \\
& =\frac{(1 / 1 \text { quintillion }) \cdot(1 /(1 \text { quintillion }+1))}{(1 / 1 \text { quintillion }) \cdot(1 /(1 \text { quintillion }+1))+1 \cdot(1 /(1 \text { quintillion }+1))} \\
& =\frac{1}{1 \text { quintillion }+1} \\
\Rightarrow P(\text { Only 42 } \mid 42) & =\frac{1 \text { quintillion }}{1 \text { quintillion }+1}
\end{aligned}
$$


the universe is directly analogous to our epistemic situation with respect to our quintillion-sided tectonic die. (Think of each face of the die as a possible rate of expansion of the early universe, or as a possible molecular configuration.) While it's perhaps possible that a fine-tuned universe, or a self-replicating molecule, was a very unlikely chancy accident, it is also possible that a fine-tuned universe and a self-replicating molecule were very likely or inevitable. And given our limited access, in both cases, to the conditions under which our data arose, it seems to me we ought to assign these hypotheses roughly equal prior weight. Hence, upon conditionalizing on our data, the hypothesis we ought to now think most likely was that life and the universe were very likely or inevitable. And, given that, it seems to me we ought to head into the laboratory, to try to figure out exactly why that should be.

All of this, recall, is presuming that agential intervention is off the tablethat we're saddled with the prejudice that the universe and our tectonic die are the result of blind physical forces. Blind physical forces, the point is, are just as likely to produce a fair die as they are to produce one loaded in some particular way.

There is a more sophisticated way of putting this point, which I will rehearse for the aficionados in the audience: Every version of the fine tuning argument that I have seen assumes the standard measure over the parameter space. The standard measure is roughly the continuous equivalent of a uniform distribution over the faces of our die. My point is that our scant evidence about the origins of the universe cannot justify high confidence that the standard measure accurately describes the parameter space. For a priori-that is, in the absence of any data-it seems equally likely that the parameter space is described by a measure which makes the observed parameter value likely or inevitable, and the latter hypothesis is massively confirmed by a single data point. Now, if we had access to 1-billion universes, and if their observed parameter values were not clustered in any obvious way-if they seemed, in some sense, uniformly drawn from the Real line-that would I think justify some confidence that the parameter space is accurately described by the standard measure. But we do not have access to 1-billion universes. A full defense of this point, however, will have to wait for another time. For now it's enough to point out that White's conclusion-that we should be content to see life and the universe as a happy accident-only follows on the strong claim that we should be antecedently confident that the standard measure is the true measure on the parameter space, or that the chance hypothesis is true, and White has given us no reason to believe that.

Here then, is a cautionary note: often when we're thinking about chance hypotheses and Bayesianism, we rely heavily on standard examples of chance mechanisms: dice, cards, urns, roulette wheels, lotteries, tornadoes, etc. But the problem with the use of these examples is that, when you confront them in a 
thought experiment, you do so with certain presuppositions. If I asked you to imagine a die, for example, and asked how many times in a row it would have to come up six before you'd be convinced it was loaded, the answer I imagine would be fairly large. Certainly, if the die came up six once, that wouldn't be enough. But all this reveals is that you presuppose, in the thought experiment, that the chance hypothesis-that the die is fair-is true. This is not unreasonable, of course, because you've presumably had a lot of experience with dice, and dice-like physical objects, and those experiences justify confidence in how those objects will behave. But that is not the case when we consider the molecular origins of life, and it is certainly not the case when we consider the conditions under which the universe arose. We do not have any evidence or experience which would justify an antecedently high confidence that a certain chance explanation accurately describes these processes. Given that, the standard examples of chance mechanisms are unhelpful as analogies, and are in fact distorting. We should not respond to a self-replicating molecule, or to a fine-tuned universe, the same way we should respond to the outcome of a single roll of a die, or a single spin of a roulette wheel, etc. ${ }^{17}$

I think that White himself makes this mistake. He imagines, as analogous to the origins of life and the universe, confronting a massive lottery, which selects ticket $\#_{4} 8579387593478$. And he writes,

No matter how confident or doubtful we are initially that the lottery is fair in that tickets are selected purely by chance, ticket $\# 48579387593478^{\prime} \mathrm{s}$ being selected gives us no reason at all to doubt this. Any investigation into the lottery mechanism that was motivated by the fact that ticket \#48579387593478 was selected would be misguided. (2007, 468)

That sounds plausible, but I contend its plausibility derives precisely from the distortion described above. If you are a Bayesian, and prior to witnessing any data, you took the chance hypothesis to be just as likely as the hypothesis that the lottery is biased in favor of any particular ticket, then you should now think it overwhelmingly likely that the lottery was biased in favor of ticket $\# 48579387593478$. And thus an investigation into the lottery mechanism-in an attempt to explain why the lottery is biased in favor of ticket \#48579387593478would not be misguided.

\section{Summary}

Allow me to sum up White's argument, and my case against it, before considering objections.

17. This point was made clear to me by Sahotra Sarkar, in conversation. 
White argues that because blind forces of nature are as likely to be biased in favor of life as they are to be biased in favor of any particular outcome, our data is equally unlikely under the hypothesis that it resulted from chance, and under the hypothesis that it resulted from those blind physical forces. It follows from this that our data does not disconfirm the chance hypothesis. White concludes that therefore "we should be quite content to ... [see] life as an extremely improbable 'happy accident.'"

I have raised two objections to this argument. First, I have argued that it is not at all clear that our data is equally likely under the chance and blind physical forces hypotheses. We can only know this if we specify a chance hypothesis, and then argue that it assigns a lesser probability to our data than the hypothesis of unknown bias. Importantly, it does not follow simply from the fact that the hypothesis of unknown bias is the hypothesis of unknown bias that therefore it assigns the same probability to our data as the chance hypothesis. Furthermore, I've offered what I think is a plausible toy probabilistic model of the origins of life, according to which our data would not be equally likely under the chance hypothesis, and the hypothesis of unknown bias.

Second, I have argued that, even if we grant that the chance hypothesis is not disconfirmed by our data, it simply does not follow that we should think, upon witnessing our data, that that hypothesis is likely to be true, nor does it follow that we should be 'quite content' to endorse it. While our data may not give us any reason to doubt the chance hypothesis, it may nevertheless give us a (very) strong reason to believe some alternative to the chance hypothesis is true. This alternative could simply be, for example, that our data were likely or inevitable. And if, antecedently, we thought the chance and the 'it was inevitable' hypotheses roughly equally likely_as it seems we should if we are uncertain about the conditions under which our data arose-then we should now take the most likely hypothesis to be that our data were inevitable. The task of the scientist, then, would be to explain why it is that our data-complex molecules, or cosmic parameters, or amplified rates of heart disease-was inevitable.

\section{Objections and Replies}

\subsection{Objection 1}

White argues that, in the absence of an available explanation of our data we should be content to endorse the hypothesis that the data improbably arose by chance. But in your reply to White, you rely heavily on the hypothesis 'the data were likely or inevitable,' which itself looks like a sort of explanation. So isn't your reply irrelevant, because White is explicit in his argument that no 
alternative explanations are available ${ }^{18}$

10.1.1. Reply

It is true that I rely on the hypothesis 'the data were likely or inevitable,' but I do not think this a problem. Were White to pursue this objection, he would face a dilemma. If his argument only applies when the hypothesis 'the data were likely or inevitable' is not an available hypothesis, then his argument is irrelevant with respect to the question of the origins of life and of the universe, because it clearly is possible that life (and the universe) were likely or inevitable. So White would either have to admit that that alternative is available-in which case he confronts my reply-or he has to contend that his argument is purely academic, and in fact irrelevant to the interesting question of how we should respond to the fact that life and the universe are improbable outcomes under the chance hypothesis.

\subsection{Objection 2}

You are reading White uncharitably. White's only goal in his (2007) was to argue that, when considering the origins of life, life's improbability is not, in itself, a reason to doubt the chance hypothesis. Thus it is consistent with White's argument that the chance hypothesis is disconfirmed by life's existence, as long as it is disconfirmed for a reason which is not the one White is objecting to. Thus the first objection-the conclusion of which is that, in the case of the origins of life, the chance hypothesis is disconfirmed-is consistent with White's argument. Furthermore, it is also perfectly consistent with White's argument that the chance hypothesis, in both your prior and posterior, is extremely unlikely. So the second objection offered in the above misses the mark as well. ${ }^{19}$

\subsubsection{Reply}

I do take White to be offering a positive argument that in fact the chance hypothesis is not disconfirmed by our data. One bit of circumstantial evidence is simply the length of his paper; that improbability does not entail disconfirmation is a point which could easily be made in a page or two. (I make it in a short paragraph in Section 2.) But more than that, White seems to me quite explicit about this in his paper; upon finishing his argument, he writes:

Where does this leave us? If life's existence is no more to be expected on the assumptions of either intentional or non-intentional biasing than it is on chance, then we have no reason to doubt the Chance hypothesis. I have been arguing that while there is at least room to argue that life

18. Thanks to a comment from an anonymous reviewer which inspired this objection. 19. Thanks to an anonymous reviewer for this objection. 
is more to be expected given that an agent was involved, it is very hard to see why we should find life's existence any more likely at all on the assumption that non-intentional biasing factors were involved. So unless we suspect that life arose on purpose, we should be quite content to join Crick in seeing life as an extremely improbable 'happy accident'. (2007 467)

Now, we might try to read White as merely arguing that the chance hypothesis is not disconfirmed by the data. In which case, while my first objection would be on target, my second objection-that, granting the chance hypothesis is not disconfirmed, the chance hypothesis could nevertheless be very unlikely in your posterior-would miss the mark, because it would be consistent with White's overall argument.

But again, I think there is pretty compelling evidence that White wants to conclude something stronger-namely, that in light of the fact that the chance hypothesis is not disconfirmed, we should think it plausibly true. White's motivating question is "why, if appeals to intelligent agency are not on the table, we should be so reluctant to attribute the origin of life largely to chance?" 2007 454). He wonders why "the vast majority of researchers in the field agree with Dawkins that we cannot credibly suppose that life arose by spontaneous random generation if the chance of this happening was extremely small?" (2007 460). He takes the upshot of his argument, as just noted, to be that we should be "quite content to join Crick in seeing life as an extremely improbable "happy accident'" 2007 467). He claims that the reasoning of researchers is "misguided" because "while making no appeal to intentional agency, [they] are persuaded that [life] was not the result of chance, and are motivated to find a non-intentional explanation" 2007 470). He claims that he will "raise doubts" about how the following two claims can "hang together" 2007, 453):

(3) "The conviction that life did not arise largely by chance is treated as epistemically prior to the development of alternative theories" (2007 453),

(4) "The suggestion that the origin of life might be due to any kind of purposeful agency is not considered as a serious option" (2007:454).

My point-that given our lack of access to the conditions under which our data arose, chance and bias hypotheses could and probably should be on equal footing in your priors, and hence should be given very little prior weightanswers the question of how these last two claims can hang together, even if the chance hypothesis is not disconfirmed by the data. Because it is reasonable to assign a low prior to the chance hypothesis, you should now be convinced, in your posterior, that life did not arise by chance. This would explain why the vast majority of researchers agree with Dawkins, why they aren't content to join 
Crick, why they are persuaded that life was not the result of chance, and why they are motivated to find a non-intentional explanation.

Finally, even if my reading of White is incorrect, and his intention was only to point out that improbability does not suffice for disconfirmation, or to argue that the chance hypothesis is not disconfirmed by the data, there would remain an interesting question: granting the chance hypothesis is not disconfirmed by their data, should scientists be motivated to search for an alternative-to-chance explanation of the origins of life and the universe? And here I've given an answer: yes, under plausible probabilistic models of their epistemic situation, and even if the chance hypothesis is not disconfirmed, the hypothesis that researchers should now think most likely is that their data were likely or inevitable, and hence they should be motivated to account for why that should be.

\subsection{Objection 3}

In the discussion of the tectonic coin of unknown bias, you point out that double heads would disconfirm the chance hypothesis, because double heads is more likely if the coin is tectonic than it is if the coin is fair. But White argues directly, in the case of the origins of life, that our evidence is equally likely under both chance and non-intentional bias hypotheses. Hence the case of the tectonic coinwhich produces two heads-is not relevant to White's argument, because it's not a case where the evidence is equally likely under chance and bias hypotheses. ${ }^{20}$

\subsubsection{Reply}

White's reason for thinking that our evidence is equally likely under the chance and non-intentional bias hypotheses is that blind physical forces are just as likely to be biased in favor of life as they are to be biased in favor of any outcome. This is also true of the tectonic coin-it is equally likely to be biased in favor of as against heads. So the tectonic coin is relevant because it reveals that you can observe a random process, which is as likely to be biased in favor of as against any particular elementary outcome, and yet receive evidence that is not equally likely under the chance and bias hypotheses. Thus my point, with the tectonic coin, is simply that White draws an illicit inference: it does not follow from the fact that non-intentional bias is as likely to favor life as to disfavor it that our evidence is equally likely under the chance and non-intentional bias hypotheses. More is required.

Recall, also, our 1,0oo paper bags full of the non-self-replicating stuff of early Earth. Suppose upon shaking them up, every single one yielded a self replicator. Suppose 1 million or 1 billion such bags produced self replicators. Or consider our San Diegans again; suppose the rate of heart disease in San Diego was 100

20. Thanks again to an anonymous reviewer for this objection. 
percent-that is, every San Diegan had heart disease. If White's inference here were successful-that is, if it followed that because blind physical forces are as likely to be biased in favor of, as against, an outcome, that therefore whatever evidence we receive is equally likely under the chance and non-intentional bias hypotheses-then no data stream could call the chance hypothesis into question (unless we are willing to attribute that data stream to an intentional agent).

That strikes me as absurd. I am right now fairly confident that heart disease in San Diego is distributed via the same chance mechanisms which distribute heart disease in the general population. But if tomorrow I learned that the rate of heart disease in San Diego was 100 percent, I would not retain that confidence. And importantly we can build plausible Bayesian models of the idea that blind physical forces are, antecedently, as likely to be biased in favor as against heart disease in San Diego which impose no such commitment.

\section{Acknowledgments}

Thanks to Cory Juhl, Sahotra Sarkar, Miriam Schoenfield, Jason Schukraft, and two anonymous referees for discussion of, and comments on, earlier drafts of this paper. Thanks especially to Sinan Dogramaci for many (continuing) hours of discussion on this topic, and comments on many earlier drafts.

\section{References}

de Duve, Christian (1991). Blueprint for a Cell: The Nature and Origin of Life. Neil Patterson.

Earman, John (1992). Bayes or Bust? A Critical Examination of Bayesian Confirmation Theory. MIT Press.

Fry, Iris (2000). The Emergence of Life on Earth. Rutgers University Press.

Hawking, Stephen (1996). The Illustrated A Brief History of Time. Random House. Hoyle, Fred and Chandra Wickramasinghe (1981). Evolution from Space. J. M. Dent and Sons.

Krulwich, Robert. Which is Greater, the Number of Sand Grains on Earth or Stars in the Sky? Retrieved from www.npr.org/sections/krulwich/2012/ 09/17/161096233/which-is-greater-the-number-of-sand-grains-on-earth-or -stars-in-the-sky

White, Roger (2007). Does Origins of Life Research Rest on a Mistake? Noûs, $41(3), 453-477$. 
